\title{
Evaluation of medical treatment in Iranian children with nephrolithiasis
}

\author{
Ehsan Valavi $^{1^{\circledR}}$, Azar Nickavar $^{2^{*}}$, Kamran Shehni Nejadpour $^{3}$, Elmira Esmizadeh $^{3 \oplus}$ \\ ${ }^{1}$ Chronic Renal Failure Research Center, Pediatric Nephrology Department, Ahvaz Jundishapur University of Medical Sciences, Ahvaz, Iran \\ ${ }^{2}$ Pediatric Nephrology Department, Iran University of Medical Sciences, Tehran, Iran \\ ${ }^{3}$ Faculty of Medicine, Ahvaz Jundishapur University of Medical Sciences, Ahvaz, Iran
}

\section{AR T I C L E I N F O}

Article Type:

Original

\section{Article History:}

Received: 9 March 2021

Accepted: 20 June 2021

Published online: 10 July 2021

\section{Keywords:}

Nephrolithiasis

Treatment

Alkalinization

Surgery

\begin{abstract}
A B S T R A C T
Introduction: Nephrolithiasis has been increasingly recognized in recent years. Urine metabolic abnormality is the main cause of renal stone in children. Therefore, identification and medical treatment of metabolic abnormalities have been suggested as an alternative approach to surgical treatments.

Objectives: This study was performed to evaluate the therapeutic effect of urine alkalinization and metabolic management in children with renal stone.

Patients and Methods: A total of 300 children (from 408 renal clinics) with nephrolithiasis were enrolled in this study. All of them were treated by supportive managements, including urine alkalinization and specific medical treatment of underlying metabolic abnormality. Improvement was defined as stone resolution, stone passage or decrease of stone dimension.

Results: Mean age at diagnosis was $28.7 \pm 2.6$ months (1-150 months). About $78.8 \%$ of patients had metabolic abnormality, of which, hypercalciuria (51.7\%) and hypocitraturia (33.4\%) were the most common causes, respectively. Resolution of renal stone occurred in $89.7 \%$ of patients after one year follow up, more in children less than 5 years $(P=0.003)$, and stones smaller than $5 \mathrm{~mm}(P<0.001)$. However, $87.5 \%$ of large stones $(5-12 \mathrm{~mm})$ improved by medical treatment.

Conclusion: Pharmacologic treatment is recommended in young children with small nephrolithiasis. Pharmacologic treatment also suggested as a primary intervention in children with uncomplicated large renal stones, and prior to invasive surgical management.
\end{abstract}

Implication for health policy/practice/research/medical education:

Management of renal stone, especially non-complicated large nephrolithiasis has been a clinical challenge to reduce surgical procedures and related complications. This study was performed to evaluate the efficacy of general and specific medical treatments in children with renal stone.

Please cite this paper as: Valavi E, Nickavar A, Shehni Nejadpour K, Esmizadeh E. Evaluation of medical treatment in Iranian children with nephrolithiasis. J Nephropharmacol. 2022;11(1):e09. DOI: 10.34172/npj.2022.09.

\section{Introduction}

Nephrolithiasis is a relatively rare disorder in children, which constitutes $2 \%-3 \%$ of all patients with renal stones (1). High concentration of urinary inhibitors (magnesium citrate) with low Bonn-Risk-Index are the main inhibitory mechanisms of renal stone formation in children (2,3). However, the incidence of nephrolithiasis has been increased globally in the last 25 years (6\%-10\%), secondary to high salt intake and low water drinking in children $(4,5)$.

About $16 \%$ of these patients have a history of recurrent nephrolithiasis, which emphasizes the appropriate prevention of new stone formation and growth of existing renal stone. Treatment of nephrolithiasis has been changed during the last three decades, with a shift toward less invasive conservative management $(4,6)$. Medical dissolution therapy has been considered a safe, well tolerated, and effective strategy with low complication rate in the previous studies $(7,8)$.

\section{Objectives}

According to a few clinical trials (4), the purpose of this study was to evaluate the efficacy of medical treatment including urine alkalinization and specific treatment of urine abnormalities.

\section{Patients and Methods}

This is a cross-sectional study on 302 children (52.6\% males) with nephrolithiasis, followed in pediatric nephrology clinics on average $28 \pm 15$ (12-48) months. 
Patients with urinary tract infection, endocrine or gastrointestinal disorders, renal impairment, vitamin D intoxication, hypercalcemia, hyperuricosuria, obstructive renal stone, and surgical uropathies were excluded from the study.

Renal stones were diagnosed by renal ultrasound in the majority of patients. Meanwhile, spiral CT scan was done for detection of small stones or ureteral calculi. Microlithiasis was defined as urinary calculi $\leq 3 \mathrm{~mm}$ and nephrolithiasis as urinary stones $>3 \mathrm{~mm}$.

\section{Study design}

A 24 hours urine collection was performed for evaluation of metabolic compounds in children with nephrolithiasis. Urine calcium was measured using ion selective electron method, and urine uric acid was calculated by the uricase method. In addition, urine citrate and oxalate were calculated by the enzymatic procedure. Cystine excretion was documented by determination of hexagonal cystine crystals or discoloration of $\mathrm{Na}$ nitroprusside. Abnormal values for urine biochemistry were as follows: hypercalciuria; urine calcium $>4 \mathrm{mg} / \mathrm{kg} / \mathrm{d}$, hyperoxaluria; urine oxalate $>0.5 \mathrm{mmol} / 1.73 \mathrm{~m}^{2}$ body surface area (BSA)/ day, hyperuricosuria; urine uric acid $>815 \mathrm{mg} / 1.73 \mathrm{~m}^{2}$ BSA/day, and hypocitraturia; urine citrate $<320 \mathrm{mg} / 1.73$ $\mathrm{m}^{2} \mathrm{BSA} /$ day and hypomagnesuria; urine $\mathrm{Mg}<88 \mathrm{mg} / 1.73$ $\mathrm{m}^{2}$ BSA (9).

Increased fluid intake, dietary salt restriction $(<2-3$ $\mathrm{mEq} / \mathrm{kg}$ in young children and 2-4 $\mathrm{g}$ in older ones), high $\mathrm{K}$ diet, $100 \%$ RDA requirement for protein and calcium, in addition to simultaneous treatment of underlying metabolic abnormality were advised in all patients. Accordingly, hydrochlorothiazide was used in children with hypercalciuria at a dose of $1-2 \mathrm{mg} / \mathrm{kg} / \mathrm{d}$. Children with hyperoxaluria were treated by limitation of oxalate rich diet and pyridoxine (10-20 mg/kg/d). Meanwhile, captopril was prescribed in all children with cystinuria $(4,10)$.

Urine alkalinization with potassium citrate solution ( $2 \mathrm{mEq} / \mathrm{L} \mathrm{K}$ ) or pills $(5 \mathrm{mEq})$ was used in patients with different variants of metabolic abnormalities at the dose of $1-2 \mathrm{mEq} / \mathrm{kg} / \mathrm{d}$ in 2-3 divided dose. Medical treatment was controlled by obtaining target urine $\mathrm{PH}$ (6.2-6.8 in calcium based stone, 7 in hyperuricosuria, more than 7 in cystinuria), and continued after 6 months of stone resolution. All patients were followed every 3-4 months by serial ultrasound for at least one year.

Medical response was considered as stone passage, complete or partial stone dissolution or decreased stone size or number. Recurrence or treatment failure was applied for patients with new stone formation or stone enlargement.

\section{Data analysis}

Statistically, continuous variables were expressed as mean \pm standard deviation (SD), and discrete variables were identified as percentage. The $\chi^{2}$ and Fisher's exact tests were used to identify significant differences between proportional nominal data. Meanwhile, numerical data were compared by the student's $t$ test. A $P$ value $<0.05$ was considered statistically significant.

\section{Results}

Mean age at diagnosis was $28.7 \pm 2.6$ months (range; $1-150$ ) months, and $80.8 \%$ were younger than 5 years old at the time of diagnosis. Gender distribution was relatively equal (M/F: 1.1/1). A family history of renal stone was found in $81.3 \%$, and $62.4 \%$ of parents were relatives. Demographic and clinical characteristics of patients are presented in Tables 1 and 2.

Metabolic abnormality was found in $78.8 \%$ of patients. Stone was unilateral in $64.9 \%$ (41\% left kidney, $24 \%$ right kidney), and bilateral in $35 \%$ of their population. Totally, nephrolithiasis was found in 408 renal units (56.37\% left kidney and $43.62 \%$ right kidney).

Renal stone was less than $5 \mathrm{~mm}$ in $59.8 \%$ and $5-12 \mathrm{~mm}$ in $40.2 \%$ of renal units. There was no statistical correlation between stone dimension with metabolic abnormalities, age and gender of patients. However, hypercalciuria $(P<$ $0.001)$, hypocitraturia $(P=0.04)$ and hypomagnesuria $(P$ $=0.001)$ were more common in children younger than 5 years old. In addition, nephrolithiasis was more common in patients with structural abnormalities $(P<0.001)$.

All cases were followed for $28 \pm 15$ months (12-48). Medical response occurred in $89.7 \%$ at the first year of follow up, $93 \%$ at 2 years, $91 \%$ at 3 years and $93 \%$ at the end of follow up, respectively. Stone free rate was $71.8 \%$ at the first year, and increased to $88.3 \%$ at 4 years of follow up (Table 3). About 26 (8\%) of stone free patients had recurrent nephrolithiasis ( 10 cases in the $2^{\text {nd }}, 13$ at $3^{\text {rd }}$ and 3 in $4^{\text {th }}$ year of follow up).

Medical response occurred in $87.5 \%$ of $5-12 \mathrm{~mm}$

Table 1. Demographic and clinical characteristics of patients

\begin{tabular}{lc}
\hline Variables & Values \\
\hline Mean age at diagnosis $(\mathrm{m})$ & $28.7 \pm 2.6(1-150)$ \\
Gender (\%) male/female & $52.6 / 47.4(1.1 / 1)$ \\
Abdominal pain & $65 \%$ \\
Dysuria & $35 \%$ \\
UTI & $30.4 \%$ \\
Nocturnal enuresis & $17.2 \%$ \\
Asymptomatic & $22.5 \%$ \\
All above & $2.3 \%$ \\
Distal RTA & $11.4 \%$ \\
Anatomical abnormalities & $6 \%$ \\
\hline Left kidney & $41 \%$ \\
Right kidney & $24 \%$ \\
\hline Bilateral & $35 \%$ \\
\hline
\end{tabular}

UTI: urinary tract infection, RTA: renal tubular acidosis. 
Table 2. Number of renal stones in each kidney

\begin{tabular}{lcc}
\hline Stone number & Right kidney (\%) & Left kidney (\%) \\
\hline 1 & $91(51.1)$ & $121(52.6)$ \\
2 & $38(21.3)$ & $53(23)$ \\
3 & $22(12.4)$ & $14(6.2)$ \\
4 & $21(11.9)$ & $28(12.2)$ \\
5 & $2(1.1)$ & $6(2.6)$ \\
$\geq 6$ & $4(2.2)$ & $8(3.4)$ \\
\hline
\end{tabular}

renal stones at the first year of follow up, compared to $90 \%$ in stones smaller than $5 \mathrm{~mm}(P<0.001)$. Therefore, resolution was more common in small stones. However, medical treatment was a reasonable option in the high percentage of large stones.

Improvement of renal stone occurred in $91.5 \%$ of children younger than 5 years at the end of first year follow up, compared to $82.3 \%$ in older children $(P=0.003)$. Therefore, young children had significantly better response to conservative medical treatment.

However, metabolic abnormality (Table 4), familial history of nephrolithiasis, gender, and unilateral or bilateral renal involvement had no significant correlation to the medical response and stone resolution.

None of our patients experienced significant complication of medical treatment, which was suggested as a safe and well tolerated modality. However, gastrointestinal discomfort was the most common complication in about $2 \%$ of children, which was improved by daily dose adjustment or drug withdrawal.

\section{Discussion}

Optimal management of renal calculi has shifted from invasive surgical procedures to low complicated pharmacologic treatments. We showed appropriate resolution of renal stones with urine alkalinization and specific management of underlying metabolic abnormalities.

Mean age at diagnosis of renal stone was $28.7 \pm 2.6$ months in our patients, which was in the average reported age of $2.5-8$ years. Totally, $80.8 \%$ of our patients were $<5$ years at the time of diagnosis, similar to $68-80 \%$ incidence in the other studies $(7,10)$.

Male predominance of nephrolithiasis (1.2-4.1) has been reported in the previous studies, with a recently rising incidence in female gender. Similarly, nephrolithiasis was relatively more common in males (1.1/1) in our study. The most common symptoms were abdominal pain, restlessness and dysuria in $65 \%$ of patients, which was in accordance with the other reports $(10,11)$.

About $81.3 \%$ of our patients had a familial history of renal stone, which has been reported in $12-75 \%$ of patients, previously. The high incidence of familial nephrolithiasis might be related to more intermarriage in our population.
Table 3. Follow up of renal stones

\begin{tabular}{lcccc}
\hline Follow up & $\begin{array}{c}\text { Total } \\
\text { kidneys }\end{array}$ & $\begin{array}{c}\text { Resolution } \\
(\%)\end{array}$ & $\begin{array}{c}\text { Partial } \\
\text { improvement (\%) }\end{array}$ & $\begin{array}{c}\text { No change } \\
\%\end{array}$ \\
\hline One year & 408 & $293(71.8)$ & $73(17.9)$ & $42(10.3)$ \\
Two years & 238 & $184(77.3)$ & $38(16)$ & $16(6.7)$ \\
Three years & 135 & $99(73.3)$ & $24(17.7)$ & $12(9)$ \\
Four years & 103 & $91(88.3)$ & $5(4.8)$ & $7(6.9)$ \\
\hline
\end{tabular}

Table 4. Improvement of renal stones based on metabolic abnormality

\begin{tabular}{lccc}
\hline Variables & Positive (\%) & Negative (\%) & P value \\
\hline Hypocitraturia & 85 & 91.6 & 0.071 \\
Cystinuria & 78.9 & 90.2 & 0.114 \\
Hypercalciuria & 89.9 & 89.5 & 0.885 \\
Hyperuricosuria & 89.3 & 89.9 & 0.857 \\
Hyperoxaluria & 86.8 & 91 & 0.192 \\
Hypomagnesuria & 88.3 & 90.1 & 0.609 \\
\hline
\end{tabular}

Around $62.4 \%$ of our parents were relatives, compared to $27 \%-68 \%$ in the other nations. Accordingly, screening has been recommended in young children with a familial history of nephrolithiasis in developing countries (12).

Bilateral nephrolithiasis has been reported in 21\%-48\% of patients $(10,12)$. About $35 \%$ of our patients had bilateral nephrolithiasis, with no significant correlation to urine metabolic abnormalities and stone resolution.

Metabolic abnormality has been considered a common cause of nephrolithiasis in children, with an incidence of $40 \%-84 \%$ (10). Additionally, $78.8 \%$ of our patients had metabolic abnormalities, with predominantly hypercalciuria (51.7\%) and hypocitraturia (33.4\%), in accordance with the other reports (11).

Therefore, identification of predisposing metabolic risk factors has been recommended in these patients for determination of the best therapeutic and preventive management (10).

Pharmacologic treatment of nephrolithiasis has been recommended in the failure of fluid and dietary modification, primary metabolic disorder, and genetic hypercalciuria with normal serum calcium. Citrate compounds, allopurinol and thiazide are effective therapeutic agents in patients with hyperuricosuria and hypercalciuria. Urine citrate is a known inhibitor of calcium stone formation by decreasing calcium excretion and saturation, prevention of crystal nucleation and formation of soluble calcium compounds. Hypocitraturia has been suggested as the most frequent metabolic risk factor of calcium based renal stone, with an incidence of $19 \%-60 \%$. Potassium citrate is an effective and standard treatment for dissolution of existing renal stones, and prevention of new stone formation in patients with hypocitraturia, normal citrate excretion, hyperoxaluria, hyperuricosuria, and cystinuria. However, monitoring of urine $\mathrm{PH}$ is recommended in calcium renal stones to 
prevent further urine alkalinization and formation of calcium phosphate crystals (1,13-15).

Sodium bicarbonate is another reasonable alternative for urine alkalinization in patients with renal insufficiency or at risk of hyperkalemia. However, inhibition of calcium reabsorption by decreasing renal $\mathrm{Na}$ loading will attenuate the beneficial effect of alkaline treatment $(7,14)$.

Stone resolution occurred in $89.7-93 \%$ of our patients after one year follow up with medical treatment, in accordance with $30 \%-100 \%$ in the other reports $(7,10,14)$. In addition, resolution was more common in children $<5$ years old, since we recommend medical treatment in young children with nephrolithiasis. Similarly, Ohkawa et al (16) reported high response to urine alkalinization at younger age.

Medical management was significantly more effective in renal stones $<5 \mathrm{~mm}$ in our study, which has been also reported in Gürgöze et al (10) study. However, improvement of non-complicated large stones, although non-significant, was an important finding in our study.

Recurrence of renal stones is lower in children, compared to adults. Different studies showed $0-19 \%$ recurrence during 16-84 months follow up $(4,15)$. Similarly, a low number $(8 \%)$ of our patients had recurrent nephrolithiasis during 4 years follow up, more in the first years.

Recurrence of nephrolithiasis has been reported in 50\% of children with metabolic abnormality, compared to $10 \%$ without risk factor, which denotes to the preventive effect of potassium citrate in recurrent calcium stone disease with hypocitraturic normocalcemia or hypercalcemia, in addition to hyperuricosuria and uric acid stone (1,14-17).

Recurrence of nephrolithiasis decreased from 0.32 to 0.17 patient/year by potassium citrate treatment in Tekin et al study (14). In addition, Tasian et al showed 53\% less stone recurrence after two years of citrate treatment (4). New stone formation and growth of residual calculus fragments decreases with potassium citrate in hypocitraturic children (18).

\section{Conclusion}

Urine alkalinization and improvement of urine metabolic abnormality are considered as effective treatments of renal stones in young children, and suggested as appropriate alternatives of invasive surgical procedures in uncomplicated large stones.

\section{Limitations of the study}

Although medical response and resolution occurred in a high number of children with large renal stones, but a relatively low number of large stones was a limitation of this study, which emphasized on further studies in the larger groups of these patients in different populations.

\section{Authors' contribution}

EV was included in preparing the concept and design of study. AN participated in preparing the draft of manuscript, and evaluating the intellectual contents. KS was the principal investigator of the study. KS and EE collected the patients' information. All authors have read and approved the content of the manuscript and confirmed the accuracy or integrity of any part of the work.

\section{Conflicts of interest}

The authors declared no conflict of interest.

\section{Ethical issues}

The research followed the tenets of the Declaration of Helsinki. The Ethics Committee of Ahvaz Jundishapur University of Medical Sciences approved this study. Accordingly, written informed consent was taken from all legal guardians and parents before any intervention. This study was extracted from M.D., thesis of Kamran Shehni Nejadpour and Elmira Esmizadeh at this university (Thesis \# GP94089). Moreover, ethical issues (including plagiarism, data fabrication, double publication) have been completely observed by the authors.

\section{Funding/Support}

None.

\section{References}

1. Mokhless IA, Sakr MA, Abdeldaeim HM, Hashad MM. Radiolucent renal stones in children: combined use of shock wave lithotripsy and dissolution therapy. Urology. 2009;73:772-5. doi: 10.1016/j.urology.2008.10.066.

2. Miyake O, Yoshimura K, Yoshioka T, Koide T, Okuyama A. High urinary excretion level of citrate and magnesium in children: potential etiology for the reduced incidence of pediatric nephrolithiasis. Urol Res. 1998;26:209-13. doi: $10.1007 / \mathrm{s} 002400050048$.

3. Laube N, Klein F, Bernsmann F, Fisang C. New diagnostic tool for individual monitoring of urolithiasis risk and other metabolic diseases. Dtsch Med Wochenschr. 2014;139:17215. doi: 10.1055/s-0034-1370196.

4. Tasian GE, Copelovitch L. Evaluation and medical management of kidney stones in children. J Urol. 2014; 192(5):1329-36. doi: 10.1016/j.juro.2014.04.108.

5. Mokhless I, Zahran AR, Youssif M, Fahmy A. Tamsulosin for the management of distal ureteral stones in children: a prospective randomized study. J Pediatr Urol. 2012;8(5):5448. doi: 10.1016/j.jpurol.2011.09.008.

6. Sas DJ, Hulsey TC, Shatat IF, Orak JK. Increasing incidence of kidney stones in children evaluated in the emergency department. J Pediatr. 2010;157(1):132-7. doi: 10.1016/j. jpeds.2010.02.004.

7. Elderwy AA, Kurkar A, Hussein A, Abozeid H, Hammodda HM, Ibraheim AF. Dissolution therapy versus shock wave lithotripsy for radiolucent renal stones in children: a prospective study. J Urol. 2014;191:1491-5. doi: 10.1016/j. juro.2013.10.060.

8. Trinchieri A, Esposito N, Castelnuovo C. Dissolution of radiolucent renal stones by oral alkalinization with potassium citrate/potassium bicarbonate. Arch Ital Urol 
Androl. 2009;81:188-91.

9. Gentili A, Ria P, Lupo A, Fabris A. Cystinic nephrolithiasis: clinical experience and new diagnostic and therapeutic perspectives. G Ital Nefrol. 2016;33:gin/33.3.5.

10. Gürgöze MK, Sarı MY. Results of medical treatment and metabolic risk factors in children with urolithiasis. Pediatr Nephrol. 2011; 26:933-7. doi: 10.1007/s00467-011-1803-3.

11. Alpay H, Ozen A, Gokce I, Biyikli N. Clinical and metabolic features of urolithiasis and microlithiasis in children. Pediatr Nephrol. 2009;24:2203-9. doi: 10.1007/s00467-0091231-9.

12. Ertan P, Tekin G, Oger N, Alkan S, Horasan GD. Metabolic and demographic characteristics of children with urolithiasis in Western Turkey. Urol Res. 2011;39:105-10. doi: 10.1007/s00240-010-0306-1.

13. Ziemba JB, Matlaga BR. Guideline of guidelines: kidney stones. BJU Int. 2015;116:184-9. doi: 10.1111/bju.13080.

14. Tekin A, Tekgul S, Atsu N, Bakkaloglu M, Kendi S. Oral potassium citrate treatment for idiopathic hypocitruria in children with calcium urolithiasis. J Urol. 2002; 168:2572-4. doi: 10.1097/01.ju.0000037968.53708.1b.

15. Moe OW, Pearle MS, Sakhaee K. Pharmacotherapy of urolithiasis: evidence from clinical trials. Kidney Int. 2011;79:385-92. doi: 10.1038/ki.2010.389.

16. Ohkawa T, Ebisuno S, Morimoto S, Yasukawa S, Sonoda T, Koide T, et al. Citrate (CG-120) therapy for urolithiasis. 1. Clinical effects. Hinyokika Kiyo. 1988;34:905-17.

17. Milose JC, Kaufman SR, Hollenbeck BK, Wolf S Jr, Hollingsworth JM. Prevalence of 24-hour urine collection in high-risk stone formers. J Urol. 2014;191:376-80. doi: 10.1016/j.juJro.2013.08.080.

18. Sarica K, Erturhan S, Yurtseven C, Yagci F. Effect of potassium citrate therapy on stone recurrence and regrowth after extracorporeal shockwave lithotripsy in children. J Endourol. 2006;20:875-9. doi: 10.1089/end.2006.20.875.

Copyright $\odot 2022$ The Author(s); Published by Society of Diabetic Nephropathy Prevention. This is an open-access article distributed under the terms of the Creative Commons Attribution License (http://creativecommons.org/licenses/by/4.0), which permits unrestricted use, distribution, and reproduction in any medium, provided the original work is properly cited. 